\title{
The 2-adic eigencurve at the boundary of weight space
}

\author{
Kevin Buzzard and L. J. P. Kilford
}

\begin{abstract}
We prove that, near the boundary of weight space, the 2-adic eigencurve of tame level 1 can be written as an infinite disjoint union of 'evenly spaced' annuli, and on each annulus the slopes of the corresponding overconvergent eigenforms tend to zero.
\end{abstract}

\section{Introduction}

Coleman and Mazur constructed in [CM98] fundamental objects called eigencurves, parametrising finite slope overconvergent normalised $p$-adic eigenforms. In [CM98] many open questions are raised, one of which is whether the slopes on every component of the eigencurve tend to zero as one approaches the boundary of weight space. The object of this paper is to prove this assertion for $\mathcal{E}$, the 2-adic eigencurve of tame level 1 (this notation will not be ambiguous because we shall not consider any other eigencurves in any detail in this paper). In fact we prove much more, explicitly computing the rigid space underlying $\mathcal{E}$ near the boundary of weight space. Note that strictly speaking this eigencurve was not constructed in [CM98], because of the running assumption $p>2$, but see [Buz05] for a construction of the eigencurve in this case (using Hecke algebras; the construction using deformation theory has not been written down for $p=2$, as far as we are aware).

The first author has in recent years been making an explicit analysis of various aspects of $\mathcal{E}$, on the basis that it should serve as a guide as to what to expect for eigencurves in general. The explicit questions about slopes in [Buz04] (which were not specific to the $p=2$ case) were initially motivated by many computations of classical modular forms of level 1 , and the action of the operator $T_{2}$ on these forms; these computations gave explicit information about $\mathcal{E}$. Answers to the questions in [Buz04] would give information about $\mathcal{E}$ and other eigencurves near the centre of weight space, where the combinatorics of the slopes involved seems very complicated (although very little is known about the geometry of the abstract rigid space underlying an eigencurve, which might turn out to be very simple). Herrick (Northwestern University) in a forthcoming PhD thesis seems to have made more conceptual and slightly more general conjectures about slopes in the central region, but the only theorem of which the authors are aware that computes all slopes at a point of weight space in this central region is the main result of the accompanying article [BC05], where the slopes in weight 0 are explicitly computed for $\mathcal{E}$.

It was the second author in his thesis [Kil02] who proved the first results indicating that nearer the boundary of weight space the story seemed much simpler. More precisely, he proved [Kil02, Theorem 3.2], that the sequence of slopes of the 2-adic overconvergent eigenforms of level 4 and odd integer weight $k \in \mathbb{Z}$ was just the arithmetic progression $0,2,4, \ldots$, independent of $k$. He also obtained results about eigenforms of level 8 , and later on dealt with some higher levels. These results led to the general belief that $\mathcal{E}$ may even be isomorphic to a disjoint union of copies of weight space, near the boundary of weight space. It is this result which we prove here.

Received 4 February 2004, accepted in final form 26 May 2004, published online 21 April 2005. 2000 Mathematics Subject Classification 11F33, $14 \mathrm{G} 22$.

Keywords: eigencurve, slopes of modular forms, overconvergent modular forms.

This journal is (C) Foundation Compositio Mathematica 2005. 


\section{K. Buzzard And L. J. P. Kilford}

We now set up some notation and state our main theorem precisely, and note a corollary. Let $K$ denote any complete extension of $\mathbb{Q}_{2}$ (that is, $K$ is a field complete with respect to a nonarchimedean valuation, containing a copy of $\mathbb{Q}_{2}$ and such that the norm on $K$ induces the standard 2-adic norm on $\mathbb{Q}_{2}$ ). Let $\mathcal{W}$ denote the open disc over $K$ with centre 0 and radius 1 . We make a slightly nonstandard definition: we define a weight to be a continuous group homomorphism $\kappa: \mathbb{Z}_{2}^{\times} \rightarrow K^{\times}$ such that $\kappa(-1)=1$. The condition $\kappa(-1)=1$ is there because throughout this paper we are working in tame level 1 , and hence there are no nonzero overconvergent modular forms of weight $\kappa$ if $\kappa(-1)=-1$. If we identify a $K$-point $w \in \mathcal{W}$ with the unique weight $\kappa: \mathbb{Z}_{2}^{\times} \rightarrow K^{\times}$such that $\kappa(5)=w+1$ then this gives a bijection between the $K$-points of $\mathcal{W}$ and the set of all weights. Note that the space we are calling $\mathcal{W}$ is the one denoted $\mathcal{W}^{+}$in [CM98], because of our condition on $\kappa(-1)$.

Note that if $k=2 \ell$ is an even integer then the function $\mathbb{Z}_{2}^{\times} \rightarrow K^{\times}$defined by $x \mapsto x^{k}$ is a weight, and this weight is denoted $k$ throughout this paper. More generally, if $\chi:(\mathbb{Z} / N \mathbb{Z})^{\times} \rightarrow K^{\times}$ is a Dirichlet character of 2-power conductor $N$, and $k$ is an integer satisfying $(-1)^{k}=\chi(-1)$, then classical modular forms of level $N$, weight $k$ and character $\chi$ are, in this optic, modular forms of weight $\kappa$ where $\kappa(x)=x^{k} \chi(x)$.

Let $\mathcal{E}$ denote the 2-adic eigencurve of tame level 1 as before. Then $\mathcal{E}$ is equipped with two canonical maps, a 'weight' map $\pi: \mathcal{E} \rightarrow \mathcal{W}$ and a map $U_{2}: \mathcal{E} \rightarrow \mathbb{G}_{m}$, sending a point $e \in \mathcal{E}$ to the $U_{2}$-eigenvalue of the corresponding finite slope eigenform. Let $s: \mathcal{E}(K) \rightarrow \mathbb{R}$ ( $s$ for slope) denote the composite of $U_{2}$ with the valuation map $v: K^{\times} \rightarrow \mathbb{R}$ (normalised so that $v(2)=1$ ).

Let $\mathcal{W}_{1}$ denote the open annulus $\{1 / 8<|w|<1\}$ of $\mathcal{W}$ and let $\mathcal{E}_{1}$ denote $\pi^{-1}\left(\mathcal{W}_{1}\right)$.

Theorem A. The rigid space $\mathcal{E}_{1}$ can be written as a countably infinite disjoint union $\mathcal{E}_{1}=\bigcup_{i=0}^{\infty} X_{i}$, and the $X_{i}$ have the following properties:

(i) $\pi: X_{i} \rightarrow \mathcal{W}_{1}$ is an isomorphism for all $i$, and

(ii) if one identifies $X_{i}$ with $\mathcal{W}_{1}$ via (i) above, then the induced map $s: \mathcal{W}_{1}(K) \cong X_{i}(K) \rightarrow \mathbb{R}$ is the map sending $w$ to $i v(w)$.

We remark that the naive generalisation of Theorem $\mathrm{A}$ to the annulus $\{1 / 8 \leqslant|w|<1\}$ is false; for example $v\left(5^{10}-1\right)=3$ but there is a classical cuspidal eigenform $q+16 q^{2}-156 q^{3}+256 q^{4}+\cdots$ of level 2, weight 10 and slope 4, and hence the slopes are not all integer multiples of 3 .

An elementary consequence of Theorem A is the following result.

Theorem B. If $\kappa$ is a weight such that $|\kappa(5)-1|>1 / 8$, then the slopes of the overconvergent modular forms of weight $\kappa$ are $\{0, t, 2 t, 3 t, \ldots\}$, where $t=v(\kappa(5)-1)$, and each slope occurs with multiplicity 1.

Note that Theorem A trivially implies Theorem B. In fact the majority of this paper is a proof of Theorem B, and in the last section we show how to deduce Theorem A from Theorem B.

Theorem $\mathrm{B}$ has consequences in the classical theory of modular forms. Let $k \geqslant 2$ be an integer (even or odd) and let $\chi$ be a Dirichlet character of conductor $N$, where $N \geqslant 4$ is a power of 2 . Assume furthermore that $(-1)^{k}=\chi(-1)$. Let $M_{k}(N, \chi)$ denote the space of classical modular forms of weight $k$, level $N$ and character $\chi$, let $S_{k}(N, \chi)$ denote the subspace of cusp forms, and let $U_{2}$ denote the classical Hecke operator on these spaces. One easily checks using Theorem 1 of [CO77] that $\operatorname{dim} M_{k}(N, \chi)=2+\operatorname{dim} S_{k}(N, \chi)=\frac{1}{8}(k-1) N+1$. Furthermore, the classical construction of Eisenstein series (see for example [Miy89, Theorems 7.1.3 and 7.2.12], and a consideration of how the Diamond operators act on the Eisenstein series constructed there) shows that the space 


\section{The 2-ADIC EIGENCURVE AT THE BOUNDARY OF WEIGHT SPACE}

of Eisenstein series in $M_{k}(N, \chi)$ is two-dimensional and has a basis consisting of the eigenforms

$$
\frac{L(1-k, \chi)}{2}+\sum_{n \geqslant 1}\left(\sum_{d \mid n} \chi(d) d^{k-1}\right) q^{n}
$$

and

$$
\sum_{n \geqslant 1}\left(\sum_{d \mid n} d^{k-1} \chi(n / d)\right) q^{n} .
$$

By the slope of an eigenform we mean the 2-adic valuation of its $U_{2}$-eigenvalue. The two Eisenstein series above have slopes 0 and $k-1$ respectively.

Corollary of Theorem B. (i) Fix an isomorphism $\mathbb{C} \cong \mathbb{C}_{2}$. Then the 2-adic valuations of the eigenvalues of $U_{2}$ on $M_{k}(N, \chi)$ are given by the set $\{0, t, 2 t, 3 t, \ldots,(k-1)\}$ where $t=8 / N$. Each valuation occurs with multiplicity one.

(ii) There are $\frac{1}{8}(k-1) N+1$ normalised eigenvectors in $M_{k}(N, \chi)$ and, when regarded as modular forms with 2-adic coefficients, each of these eigenvectors has $q$-expansion in $\mathbb{Q}_{2}(\chi)[[q]]$.

Remark. The case $N=4$ of this result is Theorem 1.1 of [Kil02].

Proof of Corollary. (i) Set $K=\mathbb{C}_{2}$. Because $\chi$ is primitive, we know that $\chi(5)$ is a primitive $(N / 4)$ th root of unity. It follows that the corresponding point $w=5^{k} \chi(5)-1$ of weight space satisfies $|w|=2^{-8 / N}$ and hence $1>|w| \geqslant 1 / 4>1 / 8$, so we may apply Theorem B to deduce that the slopes of the overconvergent forms of weight $k$ and character $\chi$ are $\{0, t, 2 t, 3 t, \ldots\}$, each with multiplicity one. The values 0 and $k-1$ correspond to the two Eisenstein series above, the slopes which are greater than $k-1$ cannot be classical (see for example the proof of Theorem 4.6.17(1) of [Miy89], especially equation (4.6.30)), and there are only $(k-1) / t-1$ slopes left, namely $\{t, 2 t, \ldots, k-1-t\}$; each of these must hence be the slope of a classical cuspidal eigenform (note that this argument gives an alternative proof of the main theorem of [Col97] for $p=2$ and $\kappa$ as above).

(ii) There is a basis of $M_{k}\left(\Gamma_{1}(N)\right)$ consisting of modular forms whose $q$-expansions have rational coefficients. By elementary group theory one deduces that $M_{k}(N, \chi)$ has a basis consisting of forms whose $q$-expansions have coefficients in $\mathbb{Q}(\chi)$. Hence the characteristic polynomial of $U_{2}$ on $M_{k}(N, \chi)$ has coefficients in $\mathbb{Q}(\chi)$. Regarded as a polynomial in $\mathbb{Q}_{2}(\chi)$ one sees from part (i) that the valuations of the roots are all distinct. Hence all the roots must have degree 1 over $\mathbb{Q}_{2}(\chi)$, because conjugates all have the same valuation. So the characteristic polynomial of $U_{2}$ factors into distinct linear factors and the corresponding one-dimensional subspaces of $M_{k}(N, \chi)$ must hence be Hecke equivariant and spanned by eigenforms.

We also note that our result implies that for $\chi$ a Dirichlet character of 2-power conductor $N \geqslant 4$, there are no cusp forms of level $N$, weight 1 and character $\chi$, because any such form would have slope at most $1-1=0$, so the eigenvalue of $U_{2}$ would be a 2-adic unit and our result implies that the only unit eigenvalues come from the Eisenstein family. On the other hand, this argument could already be extracted from Emerton's thesis [Eme98].

We finish this introduction with a brief sketch of the strategy of the proof of Theorem B. As Smithline [Smi00] observed, it is possible to 'explicitly' write down the matrix coefficients $\left(m_{i, j}\right)_{i, j \geqslant 0}$ of the $U$ operator on certain (low tame level) spaces of overconvergent modular forms of weight $\kappa$, with respect to a cleverly chosen basis; the trick is that the power series $\sum_{i, j} m_{i, j} X^{i} Y^{j}$ is the product of a relatively simple rational function of $X$ and $Y$ and a power series in $X$ only (see Lemma 11 for an explicit form of this result in our case). This latter power series depends on $\kappa$ and in our situation its coefficients are essentially those of the expansion of the overconvergent modular function $E_{\kappa} / V\left(E_{\kappa}\right)$ (with $E_{\kappa}$ an appropriate Eisenstein series) as a power series with 


\section{K. Buzzard And L. J. P. Kilford}

respect to a certain parameter $y$ on $X_{0}(4)$. The reason for the appearance of the function $E_{\kappa} / V\left(E_{\kappa}\right)$ is that it appears in 'Coleman's trick' for moving from forms of weight 0 to forms of weight $\kappa$. Sections 2-6 of this paper produce bounds for these power series coefficients (see in particular Lemma 10, part (i)), for $\kappa$ near the boundary of weight space. We remark that, almost paradoxically, these bounds are proved by a careful analysis of $E_{\kappa} / V\left(E_{\kappa}\right)$ for $\kappa$ near the centre of weight space (see Theorem 7 and its proof, which perhaps forms the technical heart of the paper). Once one has these bounds one deduces certain upper and lower bounds for the valuations of the $m_{i, j}$. The miracle is that, for $\kappa$ near the boundary of weight space, these bounds are enough to tell us precise valuations for the coefficients of the characteristic power series of $U$ (Proposition 15). This happens because our bounds are enough to ensure that in the infinite sums giving rise to each coefficient, one term has valuation strictly smaller than all of the others.

\section{The coefficients of the power series defining certain modular functions}

We start with some notation. All our rings are commutative and have a 1 . If $R$ is a ring, then we define two $R$-module homomorphisms $U$ and $V: R[[q]] \rightarrow R[[q]]$ thus:

$$
U\left(\sum_{n \geqslant 0} r_{n} q^{n}\right)=\sum_{n \geqslant 0} r_{2 n} q^{n}
$$

and

$$
V\left(\sum_{n \geqslant 0} r_{n} q^{n}\right)=\sum_{n \geqslant 0} r_{n} q^{2 n}
$$

It is an easy exercise to check that $V$ is a ring homomorphism and furthermore that $U(g V(h))=$ $h U(g)$ for $g, h \in R[[q]]$. The most common application of the latter results in this paper is the fact that if $h \in R[[q]]^{\times}$then $V(h)$ is too, and $U(g / V(h))=U(g) / h$.

We now assign notation to some standard modular forms and functions that will play a key role in what follows.

If $k \geqslant 2$ is an even integer, then the function

$$
E_{k}:=1+\frac{2}{\left(1-2^{k-1}\right) \zeta(1-k)} \sum_{n \geqslant 1}\left(\sum_{0<d \mid n, d \text { odd }} d^{k-1}\right) q^{n}
$$

(where $\zeta(s)$ is the Riemann zeta function) is a modular form of level 2 and weight $k$. Note that $U\left(E_{k}\right)=E_{k}$ and that if $k \geqslant 4$ then $E_{k}$ is just the 2-stabilised ordinary oldform associated to the standard Eisenstein series of weight $k$ and level 1. (Note however that we have dropped an Euler factor: $E_{k}$ is not the standard level-1 Eisenstein series.)

The function

$$
\Delta(q)=q \prod_{n \geqslant 1}\left(1-q^{n}\right)^{24}=q-24 q^{2}+252 q^{3}-1472 q^{4}+\cdots
$$

is a modular form of level 1 and weight 12; set

$$
f=\Delta\left(q^{2}\right) / \Delta(q)=q+24 q^{2}+300 q^{3}+2624 q^{4}+18126 q^{5}+\cdots,
$$

a modular function of level 2 giving an isomorphism $X_{0}(2) \rightarrow \mathbb{P}^{1}$ (this can be verified by observing that $f: X_{0}(2) \rightarrow \mathbb{P}^{1}$ has a simple zero at the cusp $\infty$ and no other zeros). We will make heavy use of $f$ as a parameter in $\S \S 2-5$ to measure how far various modular functions, and families of modular functions, overconverge.

Define

$$
y=\frac{\left(E_{2} / V\left(E_{2}\right)\right)-1}{24}=q-20 q^{3}+462 q^{5}-10696 q^{7}+\cdots,
$$




\section{The 2-ADIC EIGENCURVE AT THE BOUNDARY OF WEIGHT SPACE}

a modular function of level 4 giving an isomorphism $X_{0}(4) \rightarrow \mathbb{P}^{1}$ (this can be seen for example by noting (Lemma 2, part (iii)) that $f=\left(y+8 y^{2}\right) /(1-8 y)^{2}$ and hence $y: X_{0}(4) \rightarrow \mathbb{P}^{1}$ must have degree 1). We will make heavy use of $y$ as a parameter in $\S \S 6-8$ when discussing explicit formulae for entries of matrices representing the $U$ operator on spaces of modular forms.

Finally, define

$$
G_{4}=1+240 \sum_{n \geqslant 1} \sigma_{3}(n) q^{n},
$$

the Eisenstein series of level 1 and weight 4 .

A general convention in this paper is that if $F=a q+\cdots \in R[[q]]$ then $R[[F]]$ will be considered as a subring of $R[[q]]$ in the obvious way. Of course if $a$ is a unit in $R$ then $R[[F]]=R[[q]]$; for example, because $y=q+\cdots \in \mathbb{Z}[[q]]$ we see that $R[[q]]=R[[y]]$ for any ring $R$. Another fact that we use over and over again is the following power series identity.

Lemma 1. The formal binomial expansion of $(1+2 s)^{\ell}$ is in $1+2 \ell s \mathbb{Z}_{2}[[\ell, s]]$.

Proof. This follows easily from the binomial theorem and the fact that $v(n !) \leqslant n-1$ for $n \in \mathbb{Z}_{\geqslant 1}$, where $v$ denotes the 2 -adic valuation.

When we say that a result follows 'from the binomial theorem', we typically mean that it is a consequence of this lemma.

We now collect a few facts about the modular forms above.

\section{LEMMA 2.}

(i) $U(y)=0$ and $U\left(y^{2}\right)=\left(y+8 y^{2}\right) /(1+24 y)^{2}$.

(ii) For $m \in \mathbb{Z}_{\geqslant 0}$ we have $U\left(y^{2 m+1}\right)=0$ and $U\left(y^{2 m}\right)=\left(\left(y+8 y^{2}\right) /(1+24 y)^{2}\right)^{m}$.

(iii) $f=\left(y+8 y^{2}\right) /(1-8 y)^{2}, U(f)=24 f+2048 f^{2}, V(f)=y^{2} /\left(1-64 y^{2}\right)$.

(iv) $G_{4} / E_{2}^{2}=(1+256 f) /(1+64 f)$ and $V\left(G_{4}\right) / E_{2}^{2}=(1+16 f) /(1+64 f)$.

Proof. (i) We have $24 y=\left(E_{2} / V\left(E_{2}\right)\right)-1$, and hence $24 U(y)=\left(E_{2} / E_{2}\right)-1=0$. The fact that $U\left(y^{2}\right)=\left(y+8 y^{2}\right) /(1+24 y)^{2}$ could be proved theoretically by an analysis of the zeros and poles of $U\left(y^{2}\right)$, but nowadays a much simpler proof is just to observe that $U\left(y^{2}\right)$ and $\left(y+8 y^{2}\right) /(1+24 y)^{2}$ are both meromorphic functions on $X_{0}(4)$ with at worst four poles (counting multiplicities) and hence they will be equal if their $q$-expansions agree for the first few terms; on the other hand, checking that the first 100 terms of the $q$-expansions agree takes well under a second on a computer.

(ii) The fact that $U(y)=0$ implies that $y=q F\left(q^{2}\right)$ for some $F \in \mathbb{Z}[[q]]$. In particular, $y$ is an odd function of $q$ and hence so is $y^{2 m+1}$, so $U\left(y^{2 m+1}\right)=0$. On the other hand, $U\left(y^{2}\right)=U\left(q^{2} F\left(q^{2}\right)^{2}\right)=$ $q F(q)^{2}$, and so $U\left(y^{2 m}\right)=U\left(q^{2 m} F\left(q^{2}\right)^{2 m}\right)=q^{m} F(q)^{2 m}=U\left(y^{2}\right)^{m}$ and the result follows.

(iii), (iv) As in (i) these follow either by a brute force calculation of $q$-expansions, easily checked by a machine computation, or by a careful analysis of zeros and poles analogous to Lemma 2.2 of [Eme98].

We deduce some explicit results about how $U$ and $V$ affect overconvergence of modular functions (cf. [Eme98, Lemma 3.1]).

Corollary 3. Let $R$ be a commutative ring, and let $R[[2 y]]$ and so on denote the obvious subrings of $R[[q]]$. Let $r$ be $1,2,4$, or 8 . Then

(i) $R[[r f]]=R[[r y]]$ and $r f R[[r f]]=r y R[[r y]]$,

(ii) $V\left(R\left[\left[r^{2} f\right]\right]\right)=R\left[\left[r^{2} y^{2}\right]\right] \subseteq R[[r f]]$ and $V\left(r^{2} f R\left[\left[r^{2} f\right]\right]\right) \subseteq \operatorname{rff} R[[r f]]$,

(iii) $U(R[[r f]]) \subseteq R\left[\left[r^{2} f\right]\right]$ and $U(r f R[[r f]]) \subseteq r^{2} f R\left[\left[r^{2} f\right]\right]$. 


\section{K. Buzzard And L. J. P. Kilford}

Remark. It is well known (see Proposition 1 of the appendix of [BC05] and the remarks that follow) that $f$ can be used as a 'measure of supersingularity' in a neighbourhood of the ordinary locus of $X_{0}(1)$, and part (i) of this corollary shows that $y$ can too. Part (ii) (respectively (iii)) translates into well-known properties of $V$ (respectively $U$ ), namely that it decreases (respectively increases) overconvergence by a factor of 2 near the ordinary locus.

Proof of Corollary 3. (i) This follows easily from Lemma 2, part (iii), which shows that $8 f=$ $8 y+\cdots \in 8 y \mathbb{Z}_{2}[[8 y]]$ and hence that $r f=r y+\cdots \in r y \mathbb{Z}_{2}[[r y]]$.

(ii) We have that $V$ is an $R$-algebra homomorphism, continuous with respect to the $q$-adic topology, so by Lemma 2, part (iii) we have $V\left(R\left[\left[r^{2} f\right]\right]\right)=R\left[\left[V\left(r^{2} f\right)\right]\right]=R\left[\left[r^{2} y^{2}\right]\right]$. Now $R\left[\left[r^{2} y^{2}\right]\right] \subset$ $R[[r y]]=R[[r f]]$ and the last part comes from observing that, if a power series $G$ has no constant term, then neither does $V(G)$.

(iii) Note that $R[[r f]]=R[[r y]]=R\left[\left[r^{2} y^{2}\right]\right] \oplus r y R\left[\left[r^{2} y^{2}\right]\right]$. If $g \in R[[r f]]$ then write $g=g_{e}+g_{o}$ with $g_{e} \in R\left[\left[r^{2} y^{2}\right]\right]$ and $g_{o} \in r y R\left[\left[r^{2} y^{2}\right]\right]$. By Lemma 2, part (ii) we see that $U\left(g_{o}\right)=0$. By part (ii) above we can write $g_{e}=V(h)$ with $h \in R\left[\left[r^{2} f\right]\right]$. Hence $U(g)=U\left(g_{e}\right)=U(V(h))=h$ and everything now follows easily.

\section{Lemmas on powers of $E_{2}$}

Here we prove a few results about the formal power series $\left(E_{2}\right)^{\ell} \in \mathbb{Z}_{2}[[\ell, q]]$. Before we introduce this power series, let us say a little more about weight space.

We have already introduced weight space $\mathcal{W}$, the open unit disc; let $w$ be the usual parameter on this disc. We regard elements of $\mathbb{Z}_{2}[[w]]$ as functions on $\mathcal{W}$. We are also interested in some slightly bigger rings, corresponding to smaller discs in $\mathcal{W}$ : by the binomial theorem we have $5^{k}=(1+4)^{k}=$ $1+4 k+8 k(k-1)+\cdots \in \mathbb{Z}_{2}[[k]]$ and furthermore that $5^{k}-1=2(2 k+4 k(k-1)+\cdots)$ is in $4 k \mathbb{Z}_{2}[[2 k]]$, and in particular in the maximal ideal of $\mathbb{Z}_{2}[[k]]$. This gives us a natural inclusion $\mathbb{Z}_{2}[[w]] \rightarrow \mathbb{Z}_{2}[[k]]$, sending $w$ to $5^{k}-1$, and we shall henceforth regard $\mathbb{Z}_{2}[[w]]$ as a subring of $\mathbb{Z}_{2}[[k]]$ in this way. In fact one checks easily that the map $\mathbb{Z}_{2}[[w]] \rightarrow \mathbb{Z}_{2}[[k]]$ factors as

$$
\mathbb{Z}_{2}[[w]] \subset \mathbb{Z}_{2}[[w / 2]] \rightarrow \mathbb{Z}_{2}[[2 k]] \subset \mathbb{Z}_{2}[[k]],
$$

where the outer inclusions are the natural ones and the inner map is an isomorphism, corresponding geometrically to the isomorphism of discs $\{k \in K:|k|<2\} \rightarrow\{w \in K:|w|<1 / 2\}$ sending $k$ to $w=5^{k}-1$. The inverse of this isomorphism is the map $\mathbb{Z}_{2}[[2 k]] \rightarrow \mathbb{Z}_{2}[[w / 2]]$ sending $2 k$ to $2 \log (1+w) / \log (5)$.

We will be working with powers of $E_{2}$ so will be mostly concerned in the next couple of sections with even weight only. We introduce a new variable $\ell$ defined by $2 \ell=k$, and we regard $\mathbb{Z}_{2}[[k]]=$ $\mathbb{Z}_{2}[[2 \ell]]$ as a subring of $\mathbb{Z}_{2}[[\ell]]$. Note that the induced ring homomorphism $\mathbb{Z}_{2}[[w]] \rightarrow \mathbb{Z}_{2}[[\ell]]$ factors as

$$
\mathbb{Z}_{2}[[w]] \subset \mathbb{Z}_{2}[[w / 8]] \rightarrow \mathbb{Z}_{2}[[\ell]],
$$

where here the inclusion is the natural one and the map on the right is the isomorphism sending $w / 8$ to $\left(5^{2 \ell}-1\right) / 8 \in \ell \mathbb{Z}_{2}[[\ell]]$.

Our general convention will be to use italics to denote modular forms of fixed weight, and bold face to denote families of modular forms. We introduce our first family here: recall that $E_{2}=1+24 q+\cdots \in 1+24 q \mathbb{Z}[[q]]$ is the Eisenstein series of weight 2 and level 2 defined above. Define

$$
\mathbf{T}=\left(E_{2}\right)^{\ell},
$$

that is, $\mathbf{T}$ is the element $\left(E_{2}\right)^{\ell}$ of $1+8 \ell q \mathbb{Z}_{2}[[\ell, q]] \subseteq \mathbb{Z}_{2}[[\ell, q]]^{\times}$. One constructs $\mathbf{T}$ explicitly using the binomial theorem. If one evaluates $\mathbf{T}$ at $\ell=\ell_{0} \in \mathbb{Z}_{\geqslant 1}$ one gets the classical modular form $\left(E_{2}\right)^{\ell_{0}}$ of weight $2 \ell_{0}$. 
LEMMA 4.

(i) $E_{2} / V\left(E_{2}\right) \in 1+8 f \mathbb{Z}_{2}[[8 f]]$.

(ii) $\mathbf{T} / V(\mathbf{T}) \in 1+8 \ell f \mathbb{Z}_{2}[[\ell, 4 f]]$.

(iii) $U(\mathbf{T}) / \mathbf{T} \in 1+32 \ell f \mathbb{Z}_{2}[[\ell, 16 f]]$.

(iv) Let $\sigma$ denote the $\mathbb{Z}_{2}[[\ell]]$-algebra automorphism of $\mathbb{Z}_{2}[[\ell, q]]$ sending $q$ to $-q$. Then we have $\sigma(\mathbf{T}) / \mathbf{T} \in 1+16 \ell f \mathbb{Z}_{2}[[\ell, 8 f]]$.

(v) $V U(\mathbf{T}) / \mathbf{T} \in 1+8 \ell f \mathbb{Z}_{2}[[\ell, 8 f]]$.

(vi) $U(\mathbf{T}) / V U(\mathbf{T}) \in 1+8 \ell f \mathbb{Z}_{2}[[\ell, 8 f]]$.

Proof. (i) We have $E_{2} / V\left(E_{2}\right)=1+24 y$, and $24 y \in 8 f \mathbb{Z}_{2}[[8 f]]$ by Corollary 3 , part (i).

(ii) This follows immediately from the binomial theorem and part (i).

(iii) This follows by applying $U$ to part (ii), and then using Corollary 3, part (iii).

(iv) Note first that $\sigma$ fixes $\operatorname{Im}(V)$, and hence $\sigma(\mathbf{T}) / \mathbf{T}=[\sigma(\mathbf{T} / V(\mathbf{T}))] /[\mathbf{T} / V(\mathbf{T})]$. Next recall that $\mathbf{T} / V(\mathbf{T})=(1+24 y)^{\ell}$. Furthermore, by Lemma 2, part (i) we have $U(y)=0$ and hence $\sigma(y)=-y$, and so $\sigma(\mathbf{T} / V(\mathbf{T}))=(1-24 y)^{\ell}$. It suffices, therefore, to prove that

$$
((1-24 y) /(1+24 y))^{\ell} \in 1+16 \ell f \mathbb{Z}_{2}[[\ell, 8 f]]=1+16 \ell y \mathbb{Z}_{2}[[\ell, 8 y]],
$$

and this follows from the binomial theorem as the left-hand side is $(1-2(24 y /(1+24 y)))^{\ell}$.

(v) A formal $q$-expansion calculation shows that $2 V U(\mathbf{T})=\mathbf{T}+\sigma(\mathbf{T})$, so

$$
2 V U(\mathbf{T}) / \mathbf{T} \in 2+16 \ell f \mathbb{Z}_{2}[[\ell, 8 f]]
$$

by part (iv) and the result follows.

(vi) This follows from parts (iii) and (v).

\section{Eisenstein series of fixed weight}

In this section we analyse the growth of the Eisenstein series $E_{4 t}$, for $t \in \mathbb{Z}_{\geqslant 1}$. This analysis will be used in the next section to deduce corresponding bounds on the growth of the full Eisenstein family.

LEMMA 5.

(i) There exists a positive integer constant $c=c(t)$ such that $c E_{4 t} /\left(E_{2}\right)^{2 t} \in c+16 f \mathbb{Z}_{2}[[16 f]]$.

(ii) For any such $c$, we also have $c E_{4 t} / U\left(\left(E_{2}\right)^{2 t}\right) \in c+16 f \mathbb{Z}_{2}[[16 f]]$.

Proof. (i) Firstly recall that there exists an invertible sheaf $\omega^{4}$ of degree 1 on the modular curve $X_{0}(2)$, such that the sections of $\left(\omega^{4}\right)^{\otimes t}$ are modular forms of weight $4 t$. Recall that $G_{4}=1+$ $240 \sum_{n} \sigma_{3}(n) q^{n} \in \mathbb{Z}[[q]]$ is an Eisenstein series of level 1 and weight 4 ; because the space of modular forms of level 2 and weight 4 is two-dimensional, a basis for this space must be $G_{4}$ and $V\left(G_{4}\right)$. Because $X_{0}(2)$ has genus 0 we deduce that every modular form of level 2 and weight $4 t$ must be expressible as a homogeneous polynomial in $G_{4}$ and $V\left(G_{4}\right)$ of degree $t$. In particular $E_{k_{0}}$ can be written as a polynomial in $G_{4}$ and $V\left(G_{4}\right)$ of degree $t$. All of these forms are defined over $\mathbb{Q}$, and the argument above makes sense over $\mathbb{Q}$, and hence the polynomial has coefficients in $\mathbb{Q}$. Choose a positive integer $c$ such that $c E_{k_{0}}$ is a polynomial in $G_{4}$ and $V\left(G_{4}\right)$ with integral coefficients. By Lemma 2, part (iv), both $G_{4} /\left(E_{2}\right)^{2}$ and $V\left(G_{4}\right) /\left(E_{2}\right)^{2}$ are in $\mathbb{Z}_{2}[[16 f]]$, and hence $c E_{k_{0}} /\left(E_{2}\right)^{2 t} \in$ $\mathbb{Z}_{2}[[16 f]]$. Now comparing constant terms gives the result.

(ii) By specialising Lemma 4, part (iii) to $\ell=2 t$, we see that $U\left(\left(E_{2}\right)^{2 t}\right) /\left(E_{2}\right)^{2 t} \in 1+16 f \mathbb{Z}_{2}[[16 f]]$ and the result follows from this and part (i). 


\section{K. Buzzard And L. J. P. Kilford}

We can do better than this however; we can eliminate the constant $c$. Keeping the above notation, set $h=U\left(\left(E_{2}\right)^{2 t}\right) / V U\left(\left(E_{2}\right)^{2 t}\right)$ and define an endomorphism $\tilde{U}$ of $\mathbb{Z}_{2}[[q]]$ by $\tilde{U}(g)=U(g h)$.

LEMmA 6. If $t \in \mathbb{Z}_{\geqslant 1}$ then $E_{4 t} / U\left(\left(E_{2}\right)^{2 t}\right) \in 1+128 f \mathbb{Z}_{2}[[64 f]]$, and furthermore $E_{4 t} / U\left(\left(E_{2}\right)^{2 t}\right)$ is the unique element of $1+8 f \mathbb{Z}_{2}[[8 f]]$ fixed by the operator $\tilde{U}$.

Proof. We consider $\tilde{U}$ as an endomorphism of $\mathbb{Z}_{2}[[q]]=\mathbb{Z}_{2}[[f]]$. Set $N_{+}=8 f \mathbb{Z}_{2}[[8 f]]$ and note that $\left(N_{+}\right)^{2} \subseteq N_{+}$and that $h \in 1+2 N_{+}$by Lemma 4 , part (vi) specialised to $\ell=2 t$. In particular multiplication by $h$ induces an isomorphism $N_{+} \rightarrow N_{+}$. We see from Corollary 3, part (iii) that $\tilde{U}\left(N_{+}\right)=U\left(N_{+}\right) \subseteq 64 f \mathbb{Z}_{2}[[64 f]] \subset 8 N_{+}$. Furthermore, $\tilde{U}(1)=U(h) \in 1+2 U\left(N_{+}\right) \subseteq$ $1+128 f \mathbb{Z}_{2}[[64 f]] \subseteq 1+16 N_{+}$. Now if one defines $g_{1}=1$ and $g_{n+1}=\tilde{U}\left(g_{n}\right)$ for $n \geqslant 1$, then $g_{2}-g_{1} \in 16 N_{+} \subset 8 N_{+}$, and applying $\tilde{U}^{n-1}$ we deduce that $g_{n+1}-g_{n} \in 8^{n} N_{+}$. We deduce that $g=\lim _{n} g_{n}$ exists in $1+16 N_{+}$and satisfies $\tilde{U}(g)=g$. Now $g^{\prime}:=c E_{4 t} / U\left(\left(E_{2}\right)^{2 t}\right) \in c+N_{+}$ (notation as in the previous lemma) is easily checked to satisfy $\tilde{U}\left(g^{\prime}\right)=g^{\prime}$, and hence if one sets $g^{\prime \prime}:=g^{\prime}-c g \in N_{+}$then $g^{\prime \prime}=\tilde{U}\left(g^{\prime \prime}\right)$, and by repeatedly applying $\tilde{U}$ we deduce $g^{\prime \prime} \in 8^{n} N_{+}$for all $n \geqslant 1$ and hence $g^{\prime \prime}=0$. So $E_{4 t} / U\left(\left(E_{2}\right)^{2 t}\right)=g^{\prime} / c=g \in 1+16 N_{+}$. One final application of $\tilde{U}$ gets us a little more: we see $E_{4 t} / U\left(\left(E_{2}\right)^{2 t}\right)=g=\tilde{U}(g)=U(g h) \in U\left(1+2 N_{+}\right)=1+2 U\left(N_{+}\right) \subseteq 1+128 f \mathbb{Z}_{2}[[64 f]]$.

Finally, if $e$ is any element of $1+N_{+}$such that $\tilde{U}(e)=e$ then $g-e \in 8^{n} N_{+}$for all $n \geqslant 1$ and hence $e=g=E_{4 t} / U\left(\left(E_{2}\right)^{2 t}\right)$.

\section{Families of Eisenstein series}

In this section we use Lemma 6 to deduce an analogous result about the 2-adic family of Eisenstein series. We firstly recall the definition of the Eisenstein family.

By Theorem 7.10 and the remarks following Theorem 5.11 in [Was97], there is a unique meromorphic function $\zeta_{2}$ on $\mathcal{W}$ with the property that, if $k \geqslant 2$ is an even integer, then $\zeta_{2}\left(5^{(1-k)}-1\right)=$ $\left(1-2^{k-1}\right) \zeta(1-k)$. By Lemma 7.12 and the remarks following Proposition 7.9 in [Was97], we deduce that $2 / \zeta_{2} \in \mathbb{Z}_{2}[[w]]$ (our $w$ is Washington's $T$ ). We define a meromorphic function $\zeta^{*}$ on $\mathcal{W}$ by $\zeta^{*}(w)=\zeta_{2}(5 /(1+w)-1)$, and observe that $\zeta^{*}\left(5^{k}-1\right)=\left(1-2^{k-1}\right) \zeta(1-k)$ for $k \geqslant 2$ an even integer, and $2 / \zeta^{*} \in w \mathbb{Z}_{2}[[w]]$.

If $\kappa: \mathbb{Z}_{2}^{\times} \rightarrow K^{\times}$is a weight, then the formal power series

$$
E_{\kappa}:=1+\frac{2}{\zeta^{*}(\kappa)} \sum_{n \geqslant 1}\left(\sum_{0<d \mid n, d \text { odd }} \kappa(d) / d\right) q^{n}
$$

is an overconvergent 2-adic Eisenstein series of weight $\kappa$ (essentially by definition: see [CM98, $\S 2.4]$ ). If we fix $d \in \mathbb{Z}_{2}^{\times}$then the function on $\mathcal{W}$ defined by $\kappa \mapsto \kappa(d)$ is of the form $w \mapsto(1+w)^{u}$ for some $u \in \mathbb{Z}_{2}$, and hence is an element of $\mathbb{Z}_{2}[[w]]$, and one deduces from this that there is a power series $\mathbf{E} \in 1+w q \mathbb{Z}_{2}[[w, q]]$ such that for any $\kappa \in \mathcal{W}$ the specialisation of $\mathbf{E}$ to $\kappa$ is $E_{\kappa}$.

The fact that $\mathbf{E} \in 1+w q \mathbb{Z}_{2}[[w, q]]$ implies that $\mathbf{E} \in \mathbb{Z}_{2}[[w, q]]^{\times}$, and hence $V(\mathbf{E}) \in \mathbb{Z}_{2}[[w, q]]^{\times}$. Furthermore, because $y=q+\cdots \in \mathbb{Z}[[q]]$ we can regard $\mathbf{E} / V(\mathbf{E})$ as an element of $\mathbb{Z}_{2}[[w, y]]$. Write

$$
\mathbf{E} / V(\mathbf{E})=\sum_{i, j} a_{i, j} w^{i} y^{j}
$$

with $a_{i, j} \in \mathbb{Z}_{2}$. The result that the previous three sections have been building up to is the following theorem.

Theorem 7. If $j \geqslant i \geqslant 0$ then $8^{j-i} \mid a_{i, j}$.

The proof is given at the end of this section.

Remark. Results of a similar nature to the proposition were obtained by Emerton in [Eme98], and anyone who is familiar with this work will clearly see the debt which we owe it. Perhaps the idea that enables us to get a little further than Emerton's thesis is that we use $\mathbf{E} / U(\mathbf{T})$ rather than $\mathbf{E} / \mathbf{T}$ for our intermediary calculations; the point is that $\mathbf{E} / U(\mathbf{T})$ overconverges further than $\mathbf{E} / \mathbf{T}$. 


\section{The 2-ADIC EIGENCURVE AT THE BOUNDARY OF WEIGHT SPACE}

We remark also that Theorem 7 gives information on how far the function $E_{\kappa} / V\left(E_{\kappa}\right)$ overconverges for a given value of $\kappa$. This information will be crucial to us later.

The key intermediate step that we need to prove the theorem is the following proposition (cf. [Eme98, Proposition 3.12]). Regard $\mathbf{E}$ as an element of $\mathbb{Z}_{2}[[\ell, q]]=\mathbb{Z}_{2}[[\ell, f]]$, the ring where $\mathbf{T}$ lives.

Proposition 8. We have that $\mathbf{E} / U(\mathbf{T}) \in 1+64 \ell f \mathbb{Z}_{2}[[\ell, 64 f]]$.

Proof. Let $N_{+}$denote the subset $8 \ell f \mathbb{Z}_{2}[[\ell, 8 f]]$ of $\mathbb{Z}_{2}[[\ell, f]]$, and note that $\left(N_{+}\right)^{2} \subset N_{+}$. Set $H=$ $U(\mathbf{T}) / V U(\mathbf{T})$. Then $H \in 1+N_{+}$by Lemma 4, part (vi), and hence multiplication by $H$ induces an isomorphism $N_{+} \rightarrow N_{+}$. Define an endomorphism $\bar{U}$ of $\mathbb{Z}_{2}[[\ell, f]]$ by $\bar{U}(G):=U(G H)$. Then

$$
\bar{U}\left(N_{+}\right)=U\left(N_{+}\right)=\ell U\left(8 f \mathbb{Z}_{2}[[\ell, 8 f]]\right) \subseteq 64 \ell f \mathbb{Z}_{2}[[\ell, 64 f]]
$$

by Corollary 3, part (iii). In particular $\bar{U}\left(N_{+}\right)=U\left(N_{+}\right) \subset 8 N_{+}$. Also $\bar{U}(1) \in U\left(1+N_{+}\right) \subseteq 1+8 N_{+}$. This is enough to prove, via a Hensel-like argument as in Lemma 6, that there is an element $G \in 1+8 N_{+}$such that $\bar{U}(G)=G$. Indeed, if we set $G_{1}=1$ and $G_{n+1}=\bar{U}\left(G_{n}\right)$ for $n \geqslant 1$ then $G_{2}-G_{1} \in 8 N_{+}$and hence $G_{n+1}-G_{n} \in 8^{n} N_{+}$. So $G=\lim _{n} G_{n}$ exists in $1+8 N_{+}$and satisfies $\bar{U}(G)=G$. Moreover we have $G=\bar{U}(G)=U(G H) \in U\left(1+N_{+}\right)=1+U\left(N_{+}\right) \subseteq 1+64 \ell f \mathbb{Z}_{2}[[\ell, 64 f]]$. Hence it suffices to prove that $\mathbf{E} / U(\mathbf{T})=G$, which we do by checking that their specialisations to weight $k$ agree for infinitely many classical weights.

So now let $k_{0}=2 \ell_{0}=4 t \in \mathbb{Z}_{\geqslant 1}$ be a multiple of 4 . Specialising to $\ell=\ell_{0}$ gives an element $G\left(\ell_{0}\right) \in 1+8 f \mathbb{Z}_{2}[[8 f]]$ fixed by the endomorphism $\tilde{U}$ of Lemma 6 . Hence by Lemma 6 we have $G\left(\ell_{0}\right)=E_{2 \ell_{0}} / U\left(\left(E_{2}\right)^{\ell_{0}}\right)$, the specialisation of $\mathbf{E} / U(\mathbf{T})$ to weight $k_{0}$, and because there are infinitely many choices for $t \geqslant 1$ we deduce $G=\mathbf{E} / U(\mathbf{T})$. In particular, we deduce that $\mathbf{E} / U(\mathbf{T}) \in 1+$ $64 \ell f \mathbb{Z}_{2}[[\ell, 64 f]]$.

\section{Corollary 9.}

(i) $\mathbf{E} / V U(\mathbf{T}) \in 1+8 \ell f \mathbb{Z}_{2}[[\ell, 8 f]]$.

(ii) $V(\mathbf{E}) / V U(\mathbf{T}) \in 1+8 \ell f \mathbb{Z}_{2}[[\ell, 8 f]]$.

(iii) $\mathbf{E} / V(\mathbf{E}) \in 1+8 \ell f \mathbb{Z}_{2}[[\ell, 8 f]]$.

Proof. (i) This follows from Proposition 8 and Lemma 4, part (vi).

(ii) This follows from Proposition 8 and Corollary 3, part (ii).

(iii) This follows from parts (i) and (ii).

Proof of Theorem \%. We know by Corollary 9 and Corollary 3, part (i) that

$$
\mathbf{E} / V(\mathbf{E}) \in \mathbb{Z}_{2}[[\ell, 8 f]]=\mathbb{Z}_{2}[[w / 8,8 f]]=\mathbb{Z}_{2}[[w / 8,8 y]] .
$$

We also know that $\mathbf{E} / V(\mathbf{E}) \in \mathbb{Z}_{2}[[w, q]]=\mathbb{Z}_{2}[[w, y]]$. Hence if we write $\mathbf{E} / V(\mathbf{E})=\sum a_{i, j} w^{i} y^{j}=$ $\sum b_{i, j}(w / 8)^{i}(8 y)^{j}$ then $a_{i, j}, b_{i, j} \in \mathbb{Z}_{2}$. But $b_{i, j}=8^{i-j} a_{i, j}$ and the result follows.

\section{The Eisenstein family near the boundary of weight space}

Having established the bounds on the coefficients of the Eisenstein family we require, we no longer need the family $\mathbf{T}$ or the uniformiser $f$, and now we concentrate more on the family $\mathbf{E}$ and the uniformiser $y$. We also return to our complete extension $K$ of $\mathbb{Q}_{2}$. Let $\mathcal{O}$ denote the integers of $K$ and let $\mathbb{F}$ denote the residue field. As before, write $\mathbf{E} / V(\mathbf{E})=\sum_{i, j} a_{i, j} w^{i} y^{j}$. Now specialise to some 


\section{K. Buzzard And L. J. P. Kilford}

weight $w_{0} \in \mathcal{O}$ satisfying $1 / 8<\left|w_{0}\right|<1$, and let $\kappa$ denote the corresponding character. We deduce that $E_{\kappa} / V\left(E_{\kappa}\right) \in \mathcal{O}\left[\left[w_{0} y\right]\right]$. Write $E_{\kappa} / V\left(E_{\kappa}\right)=g_{\kappa}\left(w_{0} y\right)$ with $g_{\kappa} \in \mathcal{O}[[X]]$. Let $\bar{g}_{\kappa} \in \mathbb{F}[[X]]$ denote the reduction of $g_{\kappa}$ modulo the maximal ideal of $\mathcal{O}$.

LEMma 10.

(i) We have $\bar{g}_{\kappa}=\sum_{i \geqslant 0} X^{2^{i}-1}$. In particular, $\bar{g}_{\kappa}$ is independent of $\kappa$ (for $\kappa$ corresponding to $w_{0} \in \mathcal{W}$ with $\left.1 / 8<\left|w_{0}\right|<1\right)$.

(ii) If $n \in \mathbb{Z}_{\geqslant 0}$ then $a_{n, n} \in \mathbb{Z}_{2}^{\times}$if and only if $n+1$ is a power of 2 .

Proof. Fix $\kappa$ and say $g_{\kappa}=\sum c_{n} X^{n}$, with $c_{n}=c_{n}(\kappa) \in \mathcal{O}$. Specialising $\mathbf{E} / V(\mathbf{E})=\sum_{i, j} a_{i, j} w^{i} y^{j}$ to weight $w_{0}$ we see that we have $c_{j} w_{0}^{j}=\sum_{i} a_{i, j} w_{0}^{i}$ and hence

$$
c_{j}=\sum_{i} a_{i, j} w_{0}^{i-j}
$$

Now $a_{i, j} w_{0}^{i-j}$ is in the maximal ideal of $\mathcal{O}$ if $j>i$, by Theorem 7 and the fact that $\left|w_{0}\right|>1 / 8$. Also $a_{i, j} w^{i-j}$ is in the maximal ideal of $\mathcal{O}$ if $j<i$, because $a_{i, j} \in \mathbb{Z}_{2}$. Hence

$$
\bar{c}_{n}=\bar{a}_{n, n} \in \mathbb{F} \text {, }
$$

where here of course the bars denote reduction modulo the maximal ideal of $\mathcal{O}$. In particular, $\bar{c}_{n}$ is independent of the choice of $\kappa$, so $\bar{g}_{\kappa}$ is independent of $\kappa$. Moreover, this shows that parts (i) and (ii) of the lemma are equivalent. To finish the proof of the lemma, we only have to verify that $\bar{g}_{\kappa}=\sum_{i} X^{2^{i}-1}$ for one particular choice of $\kappa$. We make the choice $\kappa=\kappa_{0}$ corresponding to $w_{0}=4$, that is to modular forms of level 4 , weight 1 and nontrivial character. The corresponding Eisenstein series is

$$
E_{\kappa_{0}}=\sum_{a, b \in \mathbb{Z}} q^{a^{2}+b^{2}}
$$

and the corresponding ratio

$$
f_{0}:=E_{\kappa_{0}} / V\left(E_{\kappa_{0}}\right)=1+4 q-16 q^{3}+56 q^{5}-\cdots
$$

is a function on $X_{0}(8)$ which can be checked to satisfy $4 y f_{0}^{2}-(1+8 y) f_{0}+(1+8 y)=0$. We consider $f_{0}$ as an element of $\mathbb{Z}_{2}[[y]]$; then this last equation is an identity in $\mathbb{Z}_{2}[[y]]$, and setting $X=4 y=w_{0} y$, we deduce that the equation

$$
X g_{\kappa_{0}}(X)^{2}-(1+2 X) g_{\kappa_{0}}(X)+1+2 X=0
$$

must be an identity in $\mathcal{O}[[X]]$. Reducing modulo the maximal ideal of $\mathcal{O}$ we deduce

$$
X \bar{g}_{\kappa_{0}}(X)^{2}+\bar{g}_{\kappa_{0}}(X)+1=0
$$

in $\mathbb{F}[[X]]$. This quadratic equation is easily seen to have only one root in $\mathbb{F}[[X]]$, namely $\sum_{i \geqslant 0} X^{2^{i}-1}$, which is what we needed to prove.

\section{The $U$-operator near the boundary of weight space}

In this section we prove some results about the characteristic power series of $U$ acting on overconvergent forms of weight $\kappa$, where $\kappa$ corresponds to a point $w_{0}$ in weight space with $1 / 8<\left|w_{0}\right|<1$.

We briefly recall the definition of the spaces of overconvergent modular forms that interest us. It is well known (see [CM98, Proposition 2.2.7]) that $V\left(E_{\kappa}\right)$ is an overconvergent modular form of weight $\kappa$. One checks easily that if $c \in K$ with $1>|c|>1 / 8$ then the region of the $K$-rigid space $X_{0}(4)$ defined by $|c y| \leqslant 1$ is isomorphic to the region of $X_{0}(2)$ defined by $|c f| \leqslant 1$ and hence powers of $c y$ can be thought of as a Banach basis of a 2-adic Banach space $\mathbb{M}_{0}$ of overconvergent modular 


\section{The 2-ADIC EIGENCURVE AT THE BOUNDARY OF WEIGHT SPACE}

forms of weight 0 (this space depends on the choice of $c$, but we shall suppress the choice of $c$ from the notation). For $c$ sufficiently close to 1 , the space $V\left(E_{\kappa}\right) \mathbb{M}_{0}$ of overconvergent modular forms of weight $\kappa$ will be closed under the action of the standard Hecke operators, and the operator $U$ will be compact. This space has a Banach basis $\left\{V\left(E_{\kappa}\right)(c y)^{n}: n=0,1,2, \ldots\right\}$ and we shall analyse the characteristic power series of $U$ on this space by proving facts about the matrix representing $U$ with respect to this basis. So define $m_{i, j} \in K, i, j \geqslant 0$, by

$$
U\left(V\left(E_{\kappa}\right)(c y)^{j}\right)=V\left(E_{\kappa}\right) \sum_{i} m_{i, j}(c y)^{i} .
$$

This identity can be thought of as taking place in $K[[q]]$ or in $K[[y]]$. We analyse this matrix using the techniques of [Smi00] and [Kil02]. Recall that $g_{\kappa} \in \mathcal{O}[[X]]$ is defined by $g_{\kappa}\left(w_{0} y\right)=E_{\kappa} / V\left(E_{\kappa}\right)$.

LEMMA 11. The formal sum

$$
\sum_{i, j \geqslant 0} m_{i, j} X^{i} Y^{j}
$$

is equal to

$$
\frac{g_{\kappa}\left(\left(w_{0} / c\right) X\right)(1+(24 / c) X)^{2}}{(1+(24 / c) X)^{2}-Y^{2}\left(c X+8 X^{2}\right)} .
$$

Proof. We have

$$
\sum_{i} m_{i, j}(c y)^{i}=\left(E_{\kappa} / V\left(E_{\kappa}\right)\right) U\left((c y)^{j}\right) .
$$

Recall from Lemma 3 that $U\left(y^{j}\right)=0$ if $j$ is odd, and hence $m_{i, j}=0$ if $j$ is odd. For $j=2 t$ even we have $U\left(y^{j}\right)=\left(\left(y+8 y^{2}\right) /(1+24 y)^{2}\right)^{t}$ and hence

$$
\sum_{i} m_{i, j}(c y)^{i}=g_{\kappa}\left(w_{0} y\right)\left(c^{2}\left(y+8 y^{2}\right) /(1+24 y)^{2}\right)^{t} .
$$

This is an identity in $K[[y]]$ so substituting $X$ for $c y$ gives, for $j=2 t$,

$$
\sum_{i} m_{i, j} X^{i}=g_{\kappa}\left(w_{0} X / c\right)\left(\left(c X+8 X^{2}\right) /(1+24 X / c)^{2}\right)^{t} .
$$

Multiplying by $Y^{j}$ and summing over $j$ gives

$$
\sum_{i, j} m_{i, j} X^{i} Y^{j}=g_{\kappa}\left(w_{0} X / c\right) \sum_{t \geqslant 0}\left(\left(c X+8 X^{2}\right) Y^{2} /(1+24 X / c)^{2}\right)^{t}
$$

and summing the geometric progression on the right-hand side gives the result.

Recall that the $m_{i, j}$ are the matrix coefficients of $U$ acting on overconvergent modular forms of weight $\kappa_{0}$, with $\kappa_{0}$ corresponding to $w_{0} \in \mathcal{W}$. It is well known that the matrix $\left(m_{i, j}\right)$ is compact for $|c|<1$ sufficiently close to 1 , and in fact if $|c|>\left|w_{0}\right|$ then this can now be read off from the above lemma, using the fact that the coefficients of $g_{\kappa}$ are integral, and $w_{0} / c, 8 / c$ and $c$ all have norm less than 1.

\section{The characteristic power series of $U$ near the boundary of weight space}

Fix $w_{0}$ with $1 / 8<\left|w_{0}\right|<1$ as in the previous section, and let $\kappa$ be the corresponding weight. We wish to analyse the characteristic power series of the matrix $\left(m_{i, j}\right)_{i, j \geqslant 0}$ defined in the previous section, and we do this via Lemma 11 and the following three elementary but messy lemmas.

Fix $s$ a positive integer, and $0 \neq d \in \mathcal{O}$. Let $N=\left(n_{i, j}\right)_{0 \leqslant i, j \leqslant 2 s-1}$ be a $2 s$ by $2 s$ matrix with the property that $n_{i, j} \in d^{j} \mathcal{O}$ for all $0 \leqslant i, j \leqslant 2 s-1$ (note that we index our matrices starting from $(0,0))$. Assume that $n_{i, j}=0$ if $j$ is odd. Let $P(T)=\operatorname{det}(1-T N)=1+\cdots=\sum_{\alpha \geqslant 0} a_{\alpha} T^{\alpha} \in \mathcal{O}[T]$ 


\section{K. Buzzard and L. J. P. Kilford}

denote the 'characteristic power series' (although of course it is a polynomial) of $N$. For $0 \leqslant \beta \leqslant s$ let $T_{\beta}$ denote the $\beta$ by $\beta$ matrix whose $(i, j)$ th entry, $0 \leqslant i, j<\beta$, is $n_{2 i, 2 j} / d^{2 j} \in \mathcal{O}$.

Lemma 12. We have $a_{\alpha} / d^{\alpha(\alpha-1)} \in \mathcal{O}$, and furthermore for $\alpha \leqslant s$ we have $a_{\alpha} / d^{\alpha(\alpha-1)} \in \mathcal{O}^{\times}$if and only if $\operatorname{det}\left(T_{\alpha}\right) \in \mathcal{O}^{\times}$.

Proof. By definition of $\operatorname{det}(1-T N)$, we see that $(-1)^{\alpha} a_{\alpha}$ is the sum over $S$ of $d_{S}$, where $S$ ranges over the subsets of $\{0,1,2, \ldots, 2 s-1\}$ of size $\alpha$, and

$$
d_{S}=\sum_{\sigma: S \rightarrow S} \operatorname{sgn}(\sigma) \prod_{s \in S} n_{s, \sigma(s)} .
$$

Note first that $d_{S}=0$ if $S$ contains any odd element of $\{0,1,2, \ldots, 2 s-1\}$ and in particular $a_{\alpha}=0$ if $\alpha>s$. If $S$ contains only even elements then we see that $d^{\sum_{s \in S} s}$ divides $d_{S}$ and $\sum_{s \in S} s \geqslant \alpha(\alpha-1)$, with equality if and only if $S=S_{0}:=\{0,2,4, \ldots, 2 \alpha-2\}$. Hence $a_{\alpha}$ is a sum of multiples of $d^{\alpha(\alpha-1)}$, all but one of which are multiples of $d^{\alpha(\alpha-1)+1}$. We deduce $a_{\alpha} / d^{\alpha(\alpha-1)} \in \mathcal{O}$ and furthermore $a_{\alpha} / d^{\alpha(\alpha-1)} \in \mathcal{O}^{\times}$if and only if $d_{S_{0}} / d^{\alpha(\alpha-1)} \in \mathcal{O}^{\times}$. But it is an easy consequence of the definitions that $d_{S_{0}} / d^{\alpha(\alpha-1)}=\operatorname{det}\left(T_{\alpha}\right)$.

Our second lemma shows how to get a handle on the matrix $T_{\alpha}$ above.

Lemma 13. Define $s_{i, j} \in \mathbb{F}_{2}, 0 \leqslant i, j<\infty$, by

$$
\sum_{0 \leqslant i, j} s_{i, j} X^{i} Y^{j}=\frac{\sum_{m \geqslant 0} X^{2^{m}-1}}{1+X Y^{2}},
$$

the equality taking place in $\mathbb{F}_{2}[[X, Y]]$. Define $t_{i, j}=s_{2 i, 2 j}$ for $0 \leqslant i, j$. Then

$$
\sum_{0 \leqslant i, j} t_{i, j} X^{i} Y^{j}=\frac{1+X Y \sum_{m \geqslant 0} X^{2^{m}-1}}{1+X Y^{2}} .
$$

Proof. Define power series $A(X, Y), B(X, Y)$ in $\mathbb{F}_{2}[[X, Y]]$ by

$$
A(X, Y)=\left(\sum_{m \geqslant 0} X^{2^{m}-1}\right) /\left(1+X Y^{2}\right)
$$

and

$$
B(X, Y)=\left(1+X Y \sum_{m \geqslant 0} X^{2^{m}-1}\right) /\left(1+X Y^{2}\right) .
$$

Note that $A$ is a function of $X$ and $Y^{2}$ and so the lemma will follow if one can show that $X(A(X, Y)-$ $\left.B\left(X^{2}, Y^{2}\right)\right)$ is in $\mathbb{F}_{2}\left[\left[X^{2}, Y^{2}\right]\right]$, and this follows from an explicit calculation; we see that

$$
X\left(A(X, Y)-B\left(X^{2}, Y^{2}\right)\right)=\left(X^{2} Y^{2}+\sum_{m \geqslant 0}\left(X^{2}\right)^{2^{m}}\right) /\left(1+X^{2} Y^{4}\right) .
$$

Our final lemma will be used later on to verify an instance of the $\operatorname{condition} \operatorname{det}\left(T_{\alpha}\right) \in \mathcal{O}^{\times}$ appearing in Lemma 12. The proof uses an idea explained to us by Robin Chapman.

Lemma 14. Fix an integer $\alpha \geqslant 0$, and let $\bar{T}_{\alpha}$ be the $\alpha$ by $\alpha$ matrix $\left(\bar{t}_{i, j}\right)_{0 \leqslant i, j<\alpha}$ with entries in $\mathbb{F}_{2}$ defined via the following identity:

$$
\sum_{i, j} \bar{t}_{i, j} X^{i} Y^{j}=\frac{1+X Y\left(\sum_{m \geqslant 0} X^{2^{m}-1}\right)}{1+X Y^{2}},
$$

the equality taking place in $\mathbb{F}_{2}[X, Y] /\left(X^{\alpha}, Y^{\alpha}\right)$. Then $\operatorname{det}\left(\bar{T}_{\alpha}\right)=1$. 
The 2-ADiC EIGENCURVE AT The BOUndary OF WeIGHT SPACE

Proof. Write $\sum_{i, j} \bar{t}_{i, j} X^{i} Y^{j}=\sum_{j} f_{j}(X) Y^{j}$, with $f_{j}(X) \in V:=\mathbb{F}_{2}[X] /\left(X^{\alpha}\right)$. It suffices to prove that the $f_{j}(X), 0 \leqslant j<\alpha$, span $V$ as an $\mathbb{F}_{2}$-vector space. Write $r=\sum_{m \geqslant 0} X^{2^{m}} \in V$. Then

$$
\sum_{j} f_{j}(X) Y^{j}=(1+Y r)\left(1+X Y^{2}+X^{2} Y^{4}+X^{3} Y^{6}+\cdots\right)
$$

and one deduces, by comparing coefficients of powers of $Y$, that $f_{2 t}(X)=X^{t}$ and $f_{2 t+1}(X)=X^{t} r$. Because $r^{2}-r=X$ we see that $f_{2 t}(X)=\left(r^{2}-r\right)^{t}$ and that $f_{2 t+1}(X)=r\left(r^{2}-r\right)^{t}$, and hence that as polynomials in $r$ we have $\operatorname{deg}\left(f_{n}\right)=n$. Hence the span of the $f_{j}$ contains the image of $\mathbb{F}_{2}[r]$ in $V$. This is enough as $r=X+\cdots$ so this image is $\mathbb{F}_{2}[X]$.

We are now ready to prove the main result of this section. As usual let $\kappa$ be a weight such that the corresponding $w_{0}$ satisfies $1 / 8<\left|w_{0}\right|<1$, and let $\left(m_{i, j}\right)$ be the matrix representing $U$ in weight $\kappa$ as before.

Proposition 15. If $P_{\kappa}(T)=\sum_{\alpha \geqslant 0} b_{\alpha} T^{\alpha}$ denotes the characteristic power series of $U$ in weight $\kappa$, then $\left|b_{\alpha}\right|=\left|w_{0}\right|^{\alpha(\alpha-1) / 2}$.

Proof. If $\beta \geqslant 0$ and $M_{\beta}$ denotes the truncated matrix $\left(m_{i, j}\right)_{0 \leqslant i, j<\beta}$, and if $P_{\beta}(T)=\operatorname{det}(1-$ $\left.T M_{\beta}\right)$ is the characteristic power series of $M_{\beta}$, then the $P_{\beta}(T)$ tend to $P_{\kappa}(T)$, in the sense that if $P_{\beta}(T)=\sum_{\alpha} b_{\alpha, \beta} T^{\alpha}$ then $\lim _{\beta \rightarrow \infty} b_{\alpha, \beta}=b_{\alpha}$. Hence it suffices to prove that $\left|b_{\alpha, \beta}\right|=\left|w_{0}\right|^{\alpha(\alpha-1) / 2}$ for $\beta>2 \alpha$ and furthermore we may assume $\beta=2 s-1$ is odd. Let $N_{\beta}$ be the matrix with elements $\left(n_{i, j}\right)_{0 \leqslant i, j<\beta}$ where $n_{i, j}=m_{i, j}\left(c / w_{0}\right)^{i-j}$. Then $N_{\beta}$ is easily checked to be a conjugate of $M_{\beta}$, so $P_{\beta}(T)=\operatorname{det}\left(1-T N_{\beta}\right)$. Furthermore one easily checks that Lemma 11 implies (substituting $X$ for $w_{0} / c X$ and $Y$ for $\left.c / w_{0} Y\right)$

$$
F(X, Y):=\sum_{0 \leqslant i, j<\beta} n_{i, j} X^{i} Y^{j}=\frac{g_{\kappa}(X)\left(1+24 X / w_{0}\right)^{2}}{\left(1+24 X / w_{0}\right)^{2}-Y^{2}\left(w_{0} X+8 X^{2}\right)}
$$

Note that $F(X, Y) \in \mathcal{O}[X, Y] /\left(X^{\beta}, Y^{\beta}\right)$. Extending $K$ if necessary, we may assume that there exists $d \in K$ with $d^{2}=w_{0}$. The fact that $G(X, Y):=F(X, Y / d)$ satisfies

$$
G(X, Y)=\frac{g_{\kappa}(X)\left(1+24 X / w_{0}\right)^{2}}{\left(1+24 X / w_{0}\right)^{2}-Y^{2}\left(X+8 X^{2} / w_{0}\right)} \in \mathcal{O}[X, Y] /\left(X^{\beta}, Y^{\beta}\right)
$$

shows us that $n_{i, j} / d^{j} \in \mathcal{O}$ for all $i, j$, and the fact that $F(X, Y)$ is a function of $X$ and $Y^{2}$ implies that $n_{i, j}=0$ if $j$ is odd. We are hence in a position to apply Lemma 12 to deduce that $\left|b_{\alpha, \beta}\right| \leqslant$ $|d|^{\alpha(\alpha-1)}=\left|w_{0}\right|^{\alpha(\alpha-1) / 2}$, with equality if and only if the matrix $\left(n_{2 i, 2 j} / d^{2 j}\right)_{0 \leqslant i, j<\alpha}$ has determinant a unit. Let $T_{\alpha}$ denote this matrix, and let $\bar{T}_{\alpha}$ denote its reduction modulo the maximal ideal of $\mathcal{O}$.

Reducing $G(X, Y)$ modulo the maximal ideal of $\mathcal{O}$, it becomes

$$
\bar{G}(X, Y)=\frac{\bar{g}_{\kappa}(X)}{1+X Y^{2}} \in \mathbb{F}[X, Y] /\left(X^{\beta}, Y^{\beta}\right)
$$

and by Lemmas 10 and 13 we deduce that $\bar{T}_{\alpha}=\left(\bar{t}_{i, j}\right)_{0 \leqslant i, j<\alpha}$ with

$$
\sum_{0 \leqslant i, j<\alpha} \bar{t}_{i, j}=\frac{1+X Y \sum_{m} X^{2^{m}-1}}{1+X Y^{2}}
$$

the equality taking place in $F[[X, Y]] /\left(X^{\alpha}, Y^{\alpha}\right)$. Now Lemma 14 implies that $\operatorname{det}\left(\bar{T}_{\alpha}\right)=1$ and hence $\operatorname{det}\left(T_{\alpha}\right) \in \mathcal{O}^{\times}$. This is what we wanted.

We now have enough to prove Theorem B, which we restate below. 


\section{K. Buzzard And L. J. P. Kilford}

Theorem B (restated). If $\kappa$ is a weight corresponding to $w_{0} \in \mathcal{W}$ with $1 / 8<\left|w_{0}\right|<1$, and if $v=v\left(w_{0}\right)$, then the slopes of $U$ acting on overconvergent modular forms of weight $\kappa$ are the arithmetic progression $0, v, 2 v, 3 v, 4 v, \ldots$, each appearing with multiplicity 1 .

Proof. This follows immediately from Proposition 15 and the theory of the Newton polygon.

\section{The eigencurve near the boundary of weight space}

Theorem B is already enough to deduce the results about classical forms in the Corollary of Theorem $\mathrm{B}$ of $\S 1$, so the reader who is only interested in these does not need to read this part of the paper. In this final section we deduce Theorem A from Theorem B.

Let $A$ be a reduced affinoid algebra over $K$, and equip $A$ with the supremum norm. Let $P(T)=$ $\sum_{n \geqslant 0} a_{n} T^{n}$ be a power series with the property that $\left|a_{n}\right| \rho^{n}$ tends to zero for all $\rho \in \mathbb{R}_{>0}$. In particular $P(a)$ converges for all $a \in A$. Assume furthermore that $a_{0}=1$, and that for all $x \in \operatorname{Sp}(A)$ we have $1=\left|a_{1}(x)\right|>\left|a_{2}(x)\right|>\left|a_{3}(x)\right|>\cdots$.

Lemma 16. One can factorise $P(X)$ as

$$
P(T)=(1-v T) Q(T)
$$

with $v \in A^{\times},|v|=\left|v^{-1}\right|=1$, and $Q(T)=\sum_{n \geqslant 0} b_{n} T^{n}, b_{0}=1$, and $\left|b_{n}(x)\right|=\left|a_{n+1}(x)\right|$ for all $n \geqslant 0$ and all $x \in \operatorname{Sp}(A)$.

Proof. After replacing $K$ with a finite extension if necessary, we may choose $\rho \in K$ with $\left|a_{2}\right| \leqslant$ $|\rho|<1$. Set $u_{0}=-1 / a_{1}$ and for $n \geqslant 0$ define $u_{n+1}=u_{n}-P\left(u_{n}\right) / P^{\prime}\left(u_{n}\right)$. One proves by induction on $n$, using the fact that if $u, h \in A$ and $|u|,|h| \leqslant 1$ then $P(u+h)=P(u)+h P^{\prime}(u)+h^{2} a$ for some $a \in A$ with $|a| \leqslant 1$, that $\left|P\left(u_{n}\right)\right| \leqslant|\rho|^{2^{n}},\left|P^{\prime}\left(u_{n}\right)\right|=1$, and $\left|u_{n+1}-u_{n}\right| \leqslant|\rho|^{2^{n}}$. Let $u$ be the limit of the $u_{n}$; then $P(u)=0$, and the fact that $\left|u+1 / a_{1}\right|<1$ shows that $|u(x)|=1$ for all $x \in \operatorname{Sp}(A)$. Set $v=1 / u$ and define $P(T)\left(1+v T+v^{2} T^{2}+\cdots\right)=: Q(T)=: \sum b_{n} T^{n}$. The fact that $P(u)=0$ implies that $\left|1+a_{1} u(x)+a_{2} u^{2}(x)+\cdots+a_{n} u^{n}(x)\right|=\left|a_{n+1}(x)\right|$ for all $x \in \operatorname{Sp}(A)$ and one easily deduces from this that $\left|b_{n}(x)\right|=\left|a_{n+1}(x)\right|$ for all $n \geqslant 0$ and for all $x \in \operatorname{Sp}(A)$.

Corollary 17. If $\mathcal{X}=\operatorname{Sp}(A)$ is an affinoid subdomain of $\mathcal{W}_{1}$, with $\beta: \mathcal{X} \rightarrow \mathcal{W}_{1}$ the inclusion, and $P_{\mathcal{X}}(T) \in A[[T]]$ denotes the characteristic power series of $U$ on overconvergent modular forms of weight $\mathcal{X}$, then $P(T)=\prod_{n \geqslant 0}\left(1-\lambda_{n} T\right)$ with $\lambda_{n} \in A$ and $v\left(\lambda_{n}(x)\right)=n v(\beta(x))$.

Proof. We repeatedly apply the lemma and then substitute $P(T)$ for $Q(T / W)$, where $W \in A$ is the pullback of the parameter $w$ on $\mathcal{W}_{1}$. Proposition 15, and the fact that taking characteristic power series commutes with specialisation (see for example [Buz05, Corollary 2.9]) ensures that the conditions of the lemma are always satisfied.

Proof of Theorem A. The zero locus of $P_{\mathcal{X}}(T)$ is, by Corollary 17, a disjoint union of copies of $\mathcal{X}$, and letting $\mathcal{X}$ increase shows that the spectral curve corresponding to $U$ is isomorphic to a disjoint union of annuli over weight space and that their valuations are as predicted in Theorem A. It remains to show that the eigencurve is isomorphic to the spectral curve over $\mathcal{W}_{1}$, but this follows from the fact that the fibres of the spectral curve are reduced and hence that any overconvergent eigenform of weight in $\mathcal{W}_{1}$ is determined by its weight and its $U_{2}$-eigenvalue (see [CM98, Proposition 6.3.2]).

\section{ACKnowledgements}

The genesis of this project came from two places: firstly Emerton's thesis [Eme98], where he computes some facts about the smallest slope cuspidal family, that is the region of the eigencurve 


\section{The 2-ADiC EIGENCURVE AT THE BOUndARY OF WEIGHT SPACE}

denoted $X_{1}$ above (indeed many of the computations in $\S \S 2-5$ are variants on results in his thesis); and secondly the thesis of the second author [Kil02] (and also Smithline's thesis [Smi00]), the techniques of which are used and extended a little in $\S \S 7$ and 8. Although none of the results in this paper rely on computer calculations, ${ }^{1}$ computers were used extensively throughout the work to spot conjectures about the growth of certain power series coefficients. Our computations were done using the MAGMA computer algebra system [BCP97], and many were done on a dual processor machine running Redhat Linux purchased with a grant awarded to the first author by the University of London Central Research Fund. Most of the write-up of the first version of this paper was done during a visit to the Banff International Research Station in December 2003. These organisations are all duly thanked. We remark that the first version of this paper used a completely different set of uniformisers and only treated the region $1 / 4<|w|<1$, and it was Frank Calegari who encouraged us to extend the results to $|w|=1 / 4$ and beyond. Finally, the first author would like to thank his mother for looking after his kids for a weekend in January 2004 whilst he changed all the variables in the paper to deal with the region $1 / 8<|w| \leqslant 1 / 4$ of weight space, and as a consequence had to recalculate and rewrite almost every lemma of the paper.

\section{REFERENCES}

BCP97 W. Bosma, J. Cannon and C. Playoust, The Magma algebra system I: The user language, J. Symbolic Comput. 24 (1997), 235-265, available at http://www.maths.usyd.edu.au:8000/u/magma/.

BC05 K. Buzzard and F. Calegari, Slopes of overconvergent 2-adic modular forms, Compositio Math. 141 (2005), 591-604.

Buz04 K. Buzzard, Questions about slopes of modular forms, Astérisque 298 (2005), to appear.

Buz05 K. Buzzard, Eigenvarieties, Preprint (2005).

CM98 R. Coleman and B. Mazur, The eigencurve, in Galois representations in algebraic geometry, Durham, 1996, London Mathematical Society Lecture Note Series, vol. 254 (Cambridge University Press, Cambridge, 1998), 1-113.

CO77 H. Cohen and J. Oesterlé, Dimensions des espaces de formes modulaires, Lecture Notes in Mathematics, vol. 627 (Springer, Berlin, 1977), 69-78.

Col97 R. Coleman, Classical and overconvergent modular forms of higher level, J. Théor. Nombres Bordeaux 9 (1997), 395-403.

Eme98 M. Emerton, PhD thesis, Harvard University (1998).

Kil02 L. Kilford, PhD thesis, University of London (2002).

Miy89 T. Miyake, Modular forms (Springer, Berlin, 1989).

Smi00 L. Smithline, PhD thesis, University of California at Berkeley (2000).

Was97 L. Washington, Cyclotomic fields, second edition, Graduate Texts in Mathematics, vol. 83 (Springer, Berlin, 1997).

Kevin Buzzard buzzard@imperial.co.uk

Department of Mathematics, Imperial College, South Kensington, London SW7 2AZ, UK

L. J. P. Kilford I.kilford@gmail.com

Mathematical Institute, 24-29 St Giles', Oxford OX1 3LB, UK

\footnotetext{
${ }^{1}$ It would be tedious to prove Lemma 2 by hand, but it can be done.
} 